\title{
Aerobic Bacterial Profile and Antimicrobial Susceptibility Pattern of Pus Isolates in a South Indian Tertiary Care Hospital.
}

\author{
D.V.M.V.S.V. Raghav Rao ${ }^{1}$, Ranjan Basu ${ }^{* 2}$, Debika Roy Biswas ${ }^{3}$ \\ ${ }^{1}$ Professor \& HOD, ${ }^{* 2} M D-P G$ Resident, Final Year,,${ }^{3} M D-P G$ Resident $1{ }^{\text {st }}$ Year, \\ Dept. of Microbiology, GSL Medical College, Rajahmundry, Andhra Pradesh .
}

\begin{abstract}
Introduction: Different Studies showing consistent predictable bacterial profile in wound infections, antibiotic resistance and capacity to adapt to changing environment which render the pathogens a matter of concern as a hospital acquired infections. Hence periodical monitoring of bacterial profile and their antibiotic susceptibility pattern is important.

Objective: To determine the commonly encountered pathogens in pus samples along with their antibiotic susceptibility pattern.

Materials and Methods: This study was conducted from August, 2013 to January, 2014, in GSL Medical college central lab. Pus samples received for diagnostic microbiology was processed and identification by standard protocols. Antibiotic susceptibility test was done by Kirby Bauer disc diffusion method.

Result: Out of 114 pus samples received for culture and sensitivity in the microbiology central laboratory, 102 (89.47\%) cases yielded positive culture while $12(10.53 \%)$ cases had no aerobic growth. Among the 102 culture positive pus samples, 97 yielded pure bacterial isolates and 5 yielded mixed infection; so a total number of 107 organisms were isolated out of 102 pus samples. Among the 102 culture positive cases 60 (58.82\%) were male and $42(41.18 \%)$ were females yielding a male: female ratio of 1.43. Staphylococcus aureus was the most common isolates followed by pseudomonas aeruginosa, E. coli, K. pneumoniae, Strept. Pyogenes , S. epidermidis and proteus. Among the Gram positive isolates, vancomycin, levofloxacin and clindamycin were the most susceptible drugs whereas among the Gram negative isolates, the most susceptible drugs were piperacillin / tazobactum, levofloxacin, imipenem and amikacin .

Conclution: Changing antimicrobial resistance pose challenge in treating pyogenic infections. Appropriate and judicious selection of antibiotic would limit the emerging drug resistant strains in the future to treat these clinical conditions successfully.
\end{abstract}

Key words: antimicrobial susceptibility, pseudomonas aeruginosa, pus, pyogenic infection, resistance, staphylococcus aureus .

\section{Introduction :}

Pyogenic infection is characterized by several local inflammation, usually with pus formation, generally caused by one of the pyogenic bacteria, which can produce the accumulation of dead leukocytes and infectious agent commonly known as pus ${ }^{[1]}$.Pyogenic infections may be endogenous or exogenous . A break or abrasion in the skin can provide an entryway for these surface bacteria into the body, and they stick very well to the moist edges of a cut. The bacteria begin to multiply and extend into the cut. The body's defense mechanism includes bringing immune cells into the area to fight against the bacteria. Eventually, accumulation of these cells produces the thick whitish liquid that we call pus. Coagulase positive Staphylococcus aureus has been found to be more dominant organism in pus followed by Streptococcus ${ }^{[2]}$.

Various studies across the globe have been consistent enough to show a predictable bacterial profile in the pyogenic wound infections. This makes an important observation for a clinician who intends to start empirical treatment to his patients, while laboratory culture reports are awaited.

The present study was designed to evaluate the profile of aerobic pyogenic bacteria alongwith their susceptibility to antimicrobial agents .

\section{Materials \& Methods :}

A total number of 114 pus samples received for aerobic culture and sensitivity from different IPDs \& OPDs in Microbiology Central laboratory of GSL General Hospital , Rajahmundry during a period from August , 2013 to January, 2014 were included in the study. Informed consent was taken from the patient and ethical clearance was obtained from the institute.

Received pus samples were processed on Blood Agar, Chocolate Agar, MacConkey's Agar and Nutrient Agar media and incubated at $37^{\circ} \mathrm{C}$ under aerobic condition in incubator and the organisms were identified by biochemical reactions, Gram stain and motility test as applicable as per standard operative 
procedure. The antimicrobial susceptibility testing were done by Kirby Bauer's Disk Diffusion method and interpreted as per Clinical Laboratory Standard Institution (CLSI) guidelines ${ }^{[3] \text {. }}$

Standard antibiotics like penicillin-G (10 units), ampicillin $(10 \mathrm{mcg})$, amoxyclav $(20 / 10 \mathrm{mcg})$, piperacillin/tazobactum $(100 / 10 \mathrm{mcg})$, oxacillin $(1 \mathrm{mcg})$, vancomycin $(30 \mathrm{mcg})$, ceftriaxone $(30 \mathrm{mcg})$, cefotaxime ( $30 \mathrm{mcg}$ ), ceftazidime $(30 \mathrm{mcg}$ ), cefepime ( $30 \mathrm{mcg})$, imipenem (10 mcg), aztreonam $(30 \mathrm{mcg})$, ciprofloxacin $(5 \mathrm{mcg})$, levofloxacin $(5 \mathrm{mcg})$, co-trimoxazole $(1.25 / 23.75 \mathrm{mcg})$ gentamycin $(10$ $\mathrm{mcg})$, amikacin $(30 \mathrm{mcg})$, clindamycin $(2 \mathrm{mcg})$ and erythromycin $(15 \mathrm{mcg})^{[4]}$ were tested (HIMEDIA, MUMBAI, INDIA) .

Results obtained were analyzed by counts and percentages using MS Excell , 2007 version.

\section{Results :}

Out of 114 pus samples received for culture and sensitivity in the microbiology central laboratory, 102 $(89.47 \%)$ cases yielded positive culture while $12(10.53 \%)$ cases had no growth ( Figure-1). Among the 102 culture positive pus samples, 97 yielded pure bacterial isolates and 5 yielded mixed infection; so a total number of 107 organisms were isolated out of 102 pus samples. Among the 102 culture positive cases 60 ( 58.82\%) were male and $42(41.18 \%)$ were females yielding a male : female ratio of 1.43 (Table-1). The Department wise distribution of pus samples revealed that surgery dept. was the highest contributors (35.29\%), followed by Orthopaedics (29.42\%), Gynae \& Obs. (11.76\%), Medicine (9.80\%), Skin (7.85\%) and ENT (5.88\%) departments (Table-2) .

Among the 107 culture positive pus samples, Staphylococcus aureus was 26 ( 24.29\%), Streoptococcus pyogenes was $12(11.23 \%)$, Staphylococcus epidermidis was 10 ( 9.35\%), Pseudomonas aeruginosa was $23(21.49 \%)$, Escherichia coli was 15 ( $14.02 \%)$, Klebsiella pneumonia was $13(12.15 \%)$ and Proteus sp. was $8(7.47 \%)$ ( Table-3)

The Antibiogram of Gram Positive cocci (Table-4) revealed that the Vancomycin(100\%) was the most susceptible drug followed by Levofloxacin (76.92\%) and Oxacillin (73.07\%). Gram Negative Bacilli are susceptible to Imipenam (80\%), Aztreonam (80\%), Piperacilline+Tazobactum(80\%), Levofloxacine $(80 \%)$ (Table - 5,6 ).

Figure-1 : Pie chart showing Culture positivity among pus sample

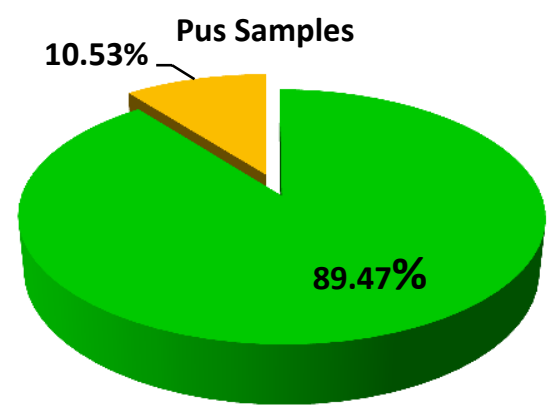

\section{Culture Positive $\quad$ Culture Negative}

Table - 1 : Sex wise distribution of culture positive pus samples

\begin{tabular}{|c|c|}
\hline Sex & $\begin{array}{c}\text { Culture Positive } \\
(\mathbf{n}=102)\end{array}$ \\
\hline Male & $\mathbf{6 0}(\mathbf{5 8 . 8 2} \%)$ \\
\hline Female & $\mathbf{4 2}(\mathbf{4 1 . 1 8 \%})$ \\
\hline Total $(\mathbf{n}=\mathbf{1 0 2})$ & $\mathbf{1 0 2}(\mathbf{1 0 0 \%})$ \\
\hline
\end{tabular}

Table - 2 : Department wise contribution of pus samples

\begin{tabular}{|c|c|c|}
\hline Serial no. & Department & Number (\%) \\
\hline 1. & Surgery & $36(35.29 \%)$ \\
\hline 2. & Orthopaedics & $30(29.42 \%)$ \\
\hline 3. & Gynae \& Obs & $12(11.76 \%)$ \\
\hline 4. & Medicine & $10(9.80 \%)$ \\
\hline 5. & Skin & $8(7.85 \%)$ \\
\hline 6. & ENT & $6(5.88 \%)$ \\
\hline \multicolumn{2}{|c|}{ Total } & $102(100 \%)$ \\
\hline
\end{tabular}


Table-3 : Aerobic bacteria ( $n=107$ ) isolated from Pus samples

\begin{tabular}{|c|c|c|}
\hline Serial No. & Organism & Number (\%) \\
\hline 1. & Staphylococcus aureus & $26(24.29 \%)$ \\
\hline 2. & Pseudomonas aeruginosa & $23(21.49 \%)$ \\
\hline 3. & Escherichia coli & $15(14.02 \%)$ \\
\hline 4. & Klebsiella pneumonia & $13(12.15 \%)$ \\
\hline 5. & Streoptococcus pyogenes & $12(11.23 \%)$ \\
\hline 6. & Staphylococcus epidermidis & $10(9.35 \%)$ \\
\hline 7. & Proteus sp & $8(7.47 \%)$ \\
\hline \multicolumn{2}{|r|}{ Total } & $107(100 \%)$ \\
\hline
\end{tabular}

Table-4 : Antibiogram of Gram Positive Cocci

\begin{tabular}{|c|c|c|c|c|c|c|}
\hline \multirow{2}{*}{$\begin{array}{c}\text { Name of } \\
\text { antibiotics }\end{array}$} & \multicolumn{2}{|c|}{ Staph. aureus $(\mathrm{n}=26)$} & \multicolumn{2}{|c|}{ Staph. epidermidis (n=10) } & \multicolumn{2}{|c|}{ Strept. pyogenes $(\mathrm{n}=12)$} \\
\hline & Sensitive & Resistance & Sensitive & Resistance & Sensitive & Resistance \\
\hline Penicillin-G & $4(15.38 \%)$ & $22(84.62)$ & $3(30 \%)$ & $7(70 \%)$ & $5(41.66 \%)$ & $7(58.34 \%)$ \\
\hline Oxacilline & 19(73.07\%) & $7(26.93 \%)$ & $\mathbf{8}(\mathbf{8 0} \%)$ & $2(20 \%)$ & $8(66.66 \%)$ & $4(33.34 \%)$ \\
\hline Vancomycin & $26(100 \%)$ & $\mathbf{0}(\mathbf{0 \%})$ & $10(100 \%)$ & $0(0 \%)$ & $12(100 \%)$ & $0(0 \%)$ \\
\hline Clindamycin & $17(65.38 \%)$ & $9(34.62 \%)$ & $7(70 \%)$ & $3(30 \%)$ & $6(50 \%)$ & $6(50 \%)$ \\
\hline Ciprofloxacin & $11(42.30 \%)$ & $15(57.7 \%)$ & $6(60 \%)$ & $4(40 \%)$ & $7(58.33 \%)$ & $5(41.67 \%)$ \\
\hline Levofloxacin & $20(76.92 \%)$ & 6(23.08\%) & $9(90 \%)$ & $1(10 \%)$ & $10(83.33 \%)$ & $2(16.67 \%)$ \\
\hline Erythromycin & $4(15.38 \%)$ & $22(84.62 \%)$ & $3(30 \%)$ & $7(70 \%)$ & $4(33.33 \%)$ & $8(66.67 \%)$ \\
\hline Gentamycin & $12(46.15 \%)$ & $14(53.85 \%)$ & $4(40 \%)$ & $6(60 \%)$ & $8(66.66 \%)$ & $4(33.34 \%)$ \\
\hline Co-trimoxazole & 3(11.53\%) & $23(88.47 \%)$ & $\mathbf{5 ( 5 0 \% )}$ & $5(50 \%)$ & $9(75 \%)$ & $3(25 \%)$ \\
\hline Tetracycline & $10(38.46 \%)$ & $16(61.54 \%)$ & $4(40 \%)$ & $6(60 \%)$ & $5(41.66 \%)$ & $7(58.34 \%)$ \\
\hline
\end{tabular}

Table-5 : Antibiogram of Enterobacteriaceae

\begin{tabular}{|c|c|c|c|c|c|c|}
\hline \multirow{2}{*}{$\begin{array}{c}\text { Name of } \\
\text { antibiotics }\end{array}$} & \multicolumn{2}{|c|}{ E. coli $(n=15)$} & \multicolumn{2}{|c|}{ Klebsiella sp. $(n=13)$} & \multicolumn{2}{|c|}{ Proteus sp. $(n=8)$} \\
\hline & Sensitive & Resistance & Sensitive & Resistance & Sensitive & Resistance \\
\hline Ampicillin & $7(46.66 \%)$ & $8(53.34 \%)$ & $6(46.15 \%)$ & $7(53.85 \%)$ & $6(75 \%)$ & $2(25 \%)$ \\
\hline Piperacillin & $10(66.66 \%)$ & $5(33.34 \%)$ & $8(61.53 \%)$ & $5(38.47 \%)$ & $5(62 \%)$ & $3(38 \%)$ \\
\hline $\begin{array}{l}\text { Piperacillin + } \\
\text { Tazobactam }\end{array}$ & $12(80 \%)$ & $3(20 \%)$ & $10(76.92 \%)$ & 3(23.08\%) & $6(75 \%)$ & $2(25 \%)$ \\
\hline Cefotaxime & $7(46.66 \%)$ & $8(53.34 \%)$ & $4(30.76 \%)$ & $9(69.24 \%)$ & $3(37 \%)$ & $5(63 \%)$ \\
\hline Ceftriaxone & $4(26.66 \%)$ & $11(73.34 \%)$ & $6(46.15 \%)$ & $7(53.84 \%)$ & $2(25 \%)$ & $6(75 \%)$ \\
\hline Ceftazidime & $10(66.66 \%)$ & $5(33.34 \%)$ & $8(61.53 \%)$ & $5(38.47 \%)$ & $3(37 \%)$ & $5(63 \%)$ \\
\hline Cefepime & $8(53.34 \%)$ & $7(46.66 \%)$ & $6(46.15 \%)$ & $7(53.84 \%)$ & $4(50 \%)$ & $4(50 \%)$ \\
\hline Imipenem & $12(80 \%)$ & $3(20 \%)$ & $10(76.92 \%)$ & $3(23.08 \%)$ & $8(100 \%)$ & $0(0 \%)$ \\
\hline Aztreonam & $12(80 \%)$ & $3(20 \%)$ & $10(76.92 \%)$ & $3(23.08 \%)$ & $7(87 \%)$ & $1(13 \%)$ \\
\hline Ciprofloxacin & $4(26.66 \%)$ & $11(73.34 \%)$ & $4(30.76 \%)$ & $9(69.24 \%)$ & $5(63 \%)$ & $3(37 \%)$ \\
\hline Levofloxacin & $12(80 \%)$ & $3(20 \%)$ & $10(76.92 \%)$ & $3(23.08 \%)$ & $7(87 \%)$ & $1(13 \%)$ \\
\hline Gentamycin & $10(66.66 \%)$ & 5(33.34\%) & $8(61.53 \%)$ & $5(38.47 \%)$ & $5(63 \%)$ & $3(37 \%)$ \\
\hline Amikacin & $12(80 \%)$ & $3(20 \%)$ & $10(76.92 \%)$ & $3(23.08 \%)$ & $6(75 \%)$ & $2(25 \%)$ \\
\hline Tobramycin & $10(66.66 \%)$ & $5(33.34 \%)$ & $8(61.53 \%)$ & $5(38.47 \%)$ & $5(63 \%)$ & $3(37 \%)$ \\
\hline
\end{tabular}

Table - 6 : Antibiogram of Pseudomonas aeruginosa

\begin{tabular}{|c|c|c|}
\hline \multirow[b]{2}{*}{ Name of antibiotics } & \multicolumn{2}{|c|}{ Pseudomonas aeruginosa $(n=23)$} \\
\hline & Sensitive & Resistance \\
\hline Ampicillin & $12(52.17 \%)$ & 11(47.83) \\
\hline Piperacillin & $15(65.21 \%)$ & $8(34.79 \%)$ \\
\hline Piperacillin+Tazobactam & $20(86.95 \%)$ & 3(13.05\%) \\
\hline Cefotaxime & $9(39.13 \%)$ & $14(60.87 \%)$ \\
\hline Ceftriaxone & $9(39.13 \%)$ & $14(60.87 \%)$ \\
\hline Ceftazidime & $9(39.13 \%)$ & $14(60.87 \%)$ \\
\hline Cefepime & $12(52.17 \%)$ & $11(47.83 \%)$ \\
\hline Imipenem & $12(52.17 \%)$ & $11(47.83 \%)$ \\
\hline
\end{tabular}

IV. Discussion :

The present study revealed S. aureus to be the most commonly occurring pathogen in pus samples which is in agreement with the studies by Tiwari P. et al ${ }^{[5]}$ and Lee C.Y. et al ${ }^{[6]}$; however, Agnihotri $\mathrm{N}$ et al ${ }^{[7]}$ found it to be second most common pathogen Pseudomonas spp Pseudomonas and E. coli spp. were the most 
common Gram Negative Bacilli (GNB) isolated from the pus samples in our study. Such GNB dominance in the aerobic growth in pus culture has been highly seconded by studies reported by Ghosh $\mathrm{A}$ et al ${ }^{[8]}$ and Zubair M. et $\mathrm{al}^{[9]}$. Another study by Basu S. et al ${ }^{[10]}$ also reported Pseudomonas and E. coli spp. to be the most commonly occurring pathogens in wound infections, in that order.

The present study revealed that the male : female distribution of pus isolates to be $1.43: 1$ which closely corroborates with the study by Pappu A.K. et al. ${ }^{[11]}$ The Department wise distribution of pus samples revealed that surgery dept. was the highest contributors (35.29\%), followed by Orthopaedics (29.42\%), Gynae \& Obs. (11.76\%), Medicine (9.80\%), Skin (7.85\%) and ENT (5.88\%) departments .The Antibiogram of Gram Positive cocci revealed that the Vancomycin $(100 \%)$ was the most susceptible drug followed by Levofloxacin $(76.92 \%)$ and Oxacillin (73.07\%). Gram nagetive Bacilli are susceptible to Imipenam (80\%), Aztreonam (80\%), Piperacilline+Tazobactum( $80 \%)$, Levofloxacine $(80 \%)$; all these observation was in agreement with the study by $\mathrm{G}$ Suguneswari et $\mathrm{al}^{[12]}$.

\section{Conclusion :}

Pyogenic infection has been the major cause of morbidity since long. Emerging multidrug resistant strains is of major concern to treat these conditions. Even though gram negative bacteria are being increased significantly but still Staphylococcus aureus is being continued as a major etiological agent of pyogenic infections. Changing antimicrobial resistance pose challenge in treating these conditions. Appropriate and judicious selection of antibiotic by using antibiotic sensitivity data would limit the emerging drug resistant strains in the future to treat these clinical conditions successfully. Our study thereby will guide the clinician in choosing appropriate antibiotics which not only contribute to better treatment but there judicious use will also help in preventing emergence of resistance to the drug which are still sensitive .

\section{References:}

[1]. Koneman, W.K., Allen, S. D., Janda, W.M., Schreckenberger, P.C., Propcop, G.W., Woods, G.L. and Winn, W.C., Jr. Philadelphia Color Atlas and Textbook of Diagnostic Microbiology, 6th ed. Lippincott-Raven Publisher, 2005, pp: 624-662.

[2]. Chopra, A., Puri, R., Mittal, R. R. and Kanta, S. 1994. A clinical and bacteriological study of pyodermas. Indian J. Dermatology, Vernology and Leprology. 60: 200-202.

[3]. Clinical and Laboratory Standard Institute ; Performance standards for antimicrobial susceptibility testing; Clinical and Laboratory Standards Institute, Wayne; $2012 ;$ 22nd Informational Supplement : 32(3).

[4]. Betty A. Forbes, Daniel F. Sahm, Alice S. Weissfeld ; Bailey \& Scott's Diagnostic Microbiology ; $12^{\text {th }}$ edition ; $2007: \mathrm{p}-210$.

[5]. Tiwari P, Kaur S. Profile and sensitivity pattern of bacteria isolated from various cultures in a Tertiary Care Hospital in Delhi. Indian J Public Health. 2010 Oct-Dec; 54(4):213-5.

[6]. Lee CY, Chen PY, Huang FL, Lin CF. Microbiologic spectrum and susceptibility pattern of clinical isolates from the pediatric intensive care unit in a single medical center - 6 years' experience. J Microbiol Immunol Infect. 2009 Apr; 42(2):160-5.

[7]. Agnihotri N, Gupta V, Joshi RM. Aerobic bacterial isolates from burn wound infections and their antibiograms--a five-year study. Burns. 2004 May; 30(3):241-3.

[8]. 8). Ghosh A, Karmakar PS, Pal J, Chakraborty N, Debnath NB, Mukherjee JD.;Bacterial incidence and antibiotic sensitivity pattern in moderate and severe infections in hospitalized patients. J Indian Med Assoc. 2009 Jan; 107(1):21-2, 24-5.

[9]. Zubair M, Malik A, Ahmad J. Clinico-microbiological study and antimicrobial drug resistance profile of diabetic foot infections in North India. Foot 2011 Mar; 21(1):6-14. Epub 2010 Dec 4.

[10]. Basu S, Ramchuran Panray T, Bali Singh T, Gulati AK, Shukla VK. A ; prospective, descriptive study to identify the microbiological profile of chronic wounds in outpatients. Ostomy Wound Manage. 2009 Jan; 55(1):14-20.

[11]. Asha Konipparambil Pappu, Aprana Sinha, Aravind Johnson,Microbiological profile of Diabetic foot ulcer. Calicut Medical Journal 2011; 9(3):e2

[12]. G. Suguneswari, A. Heraman Singh , Ranjan Basu bacteriological profile of osteomyelitis in a tertiary care hospital at Visakhapatnam.IJCRR; $2013 ; 05(20)$. 